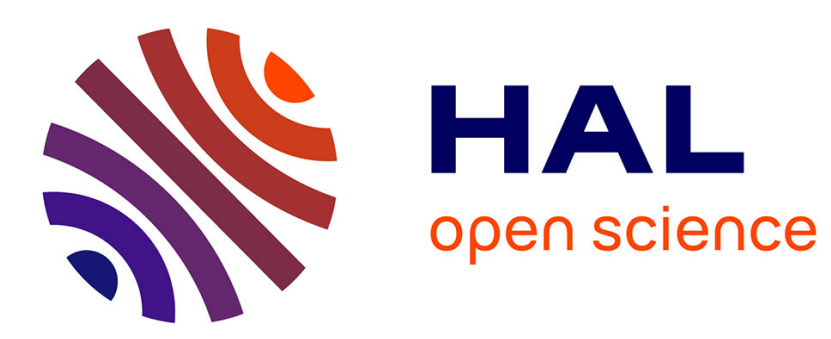

\title{
De Jérusalem à Damas: les imaginaires nationalistes de la gauche jordanienne
}

\author{
Nicolas Dot-Pouillard
}

\section{To cite this version:}

Nicolas Dot-Pouillard. De Jérusalem à Damas: les imaginaires nationalistes de la gauche jordanienne. Confluences Méditerranée, 2016, Partis et partisans dans le monde arabe post-2011, 98, pp 145-157. halshs-01383358

\section{HAL Id: halshs-01383358 https://shs.hal.science/halshs-01383358}

Submitted on 18 Oct 2016

HAL is a multi-disciplinary open access archive for the deposit and dissemination of scientific research documents, whether they are published or not. The documents may come from teaching and research institutions in France or abroad, or from public or private research centers.
L'archive ouverte pluridisciplinaire HAL, est destinée au dépôt et à la diffusion de documents scientifiques de niveau recherche, publiés ou non, émanant des établissements d'enseignement et de recherche français ou étrangers, des laboratoires publics ou privés. 
Nicolas Dot-Pouillard, De Jérusalem à Damas : les imaginaires nationalistes de la gauche jordanienne

Chercheur associé à l'Institut français du Proche-Orient, membre du programme WAFAW (WhenAuthoritarianismFails in the Arab World) de l'ERC. Il a coécrit De la théologie à la libération? Histoire du Jihad islamique palestinien (avec WissamAlhaj et Eugénie Rébillard, La Découverte, 2014) et Tunisie : la révolution et ses passés, (L'Harmattan, coll. Les cahiers de l'IReMMO, 2013)' ${ }^{1}$.

Résumé : L'histoire de la gauche jordanienne est indissociable de celle de la gauche palestinienne. Depuis le début des années 1990, elle s'est pourtant nationalisée. Active dans les mouvements sociaux et syndicaux,membre de l'opposition démocratique, elle a participé en 2011 aux mouvements de contestation de la monarchie hachémite. Certaines de ses composantes se sont rapprochées du gouvernement, tandis que d'autres adoptent une rhétorique de plus en plus nationaliste - s'appuyant sur les tribus de l'est du pays. Un calendrier proprement jordanien s'est imposé. Pourtant, la gauche a encore des agendas transnationaux. Elle reste tournée vers Jérusalem et regarde de plus en plus vers Damas. Les spectres nationalistes arabes - ou machrékiens - la taraudent. Entre utopies transnationales et acceptation de l'État-nation, la gauche jordanienne a ses horizons d'attente conflictuels.

Le 14 janvier 2011, alors que le président Zin al-Abidin Ben Ali fuit la Tunisie, le mouvement Jayyin(«nous arrivons») manifeste à Amman: il est composé de travailleurs du service public, d'activistes de gauche et d'étudiants ${ }^{2}$. Il ne demande pas la «chute du régime», et n'appelle pas au renversement de la monarchie ; tout au plus veut-il de profondes réformes démocratiques, assorties de mesures contre la «corruption» et la vie chère. Deux mois plus tard, une éphémère Coalition du 24 mars prend le relais de la contestation.

L'analogie avec la Tunisie de Ben Ali ou l'Égypte de Hosni Moubarak est pourtant inopérante. Certes, le court cycle de contestation jordanien s'inscrit dans l'onde de choc des soulèvements arabes. Mais le printemps jordanien n'a pas eu lieu.La monarchie met en place un Comité de dialogue national, incluant des figures de l'opposition, notamment de gauche, dès le mois de mars 2011. Elle tente de juguler la contestation sociale en proposant un plan d'urgence sociale, baissant notamment les taxes sur l'essence. L'exemple jordanien interroge un véritable paradoxe des processus révolutionnaires arabes. C'est l'anti-1793 : le roi n'est pas la cible. Des modèles monarchiques et républicains, les premiers sont parfois plus résilients face

\footnotetext{
${ }^{1}$ Cet article est le fruit de recherches de terrain menées avec le soutien du programme ERCWafaw (WhenAuthoritarianismfails in the Arab World-EuropeanResearch Council). Il reflète les vues de l'auteur, et non de l'institution qui l'a financé.

2 International Crisis Group, «PopularProtests in NorthAfrica and the Middle East: DallyingwithReform in Jordan », Middle East/NorthAfrica Report118, mars2012.
} 
aux mouvements sociaux : ce fut le cas de la Jordanie ${ }^{3}$. C'est l'avantage des «deux corps du roi ${ }^{4}$ : sa souveraineté ne se confond pas totalement avec le régime qui le porte.

\section{Une gauche nationaliste}

Le «printemps jordanien » fut aussi celui d'une gauche jordanienne dont les rapports à la monarchie hachémite furent toujours contrariés. Particulièrement mobilisée en 2011, composante d'une opposition démocratique aux côtés des islamistes, elle a pourtant fait retraite. Une partie de la gauche jordanienne a fait allégeance au monarque : en août 2013, Khaled Kalaldeh est nommé ministre des Affaires parlementaires, dans le gouvernement d'Abdallah Ensour. Membre fondateur du Mouvement de la gauche sociale (harakat al-yassar al-ijtima'i), ancien combattant des guerres du Liban au sein du Front démocratique pour la libération de la Palestine (FDLP) et des ansar (partisans) ${ }^{5}$, il était pourtant l'un des porte-paroles du mouvement de contestation de 2011.

La gauche jordanienne se dit révolutionnaire et puise dans le patrimoine idéologique du marxisme. Il est d'inspiration soviétique dans le cas du Parti communiste jordanien (PCJ), né en 1951,ou se teinte de coloration nationaliste arabe dans celui du Parti de l'unité populaire démocratique (Al-Wahda),issu du Front populaire pour la libération de la Palestine (FPLP) ${ }^{6}$. Mais, à l'instar du Front d'action islamique (FAI) - la branche jordanienne des Frères musulmans -, la gauche est sans illusion révolutionnaire : la monarchie ne peut être renversée. Elle se sait faible, n'ayant plus le vent idéologique en poupe, au contraire des islamistes. Ses militants sont pour l'essentiel des fonctionnaires, des étudiants, ou des employés de la classe moyenne dans le secteur privé. La gauche conservetout de même quelques bastions syndicaux.

La catégorie «gauche » doit être interrogée ; elle n'est pas chimiquement pure. Certes, il y a des airs de famille : en 2013, la création de l'Union des communistes jordaniens regroupe le Parti communiste, le Mouvement de la gauche sociale et des personnalités de gauche indépendantes ${ }^{7}$. Mais il n'est pas anodin que cette gauche côtoie, dans

\footnotetext{
${ }^{3}$ Alain Dieckhoff, "The Resilience of Arab Monarchies and the ArabSpring: a Comparative Approach », in Eberhardt Kienle and Nadine Sika (dir.), The ArabUprisings. Transforming and Challenging State Power, Londres, I.B. Tauris, 2015, p. 115.

${ }^{4}$ Ernst HartwigKantorowicz, Les deux corps du roi, Paris, Gallimard, 1989.

${ }^{5}$ Les ansar ont été fondés en 1970, sous l'impulsion du secrétaire général du Parti communiste jordanien, Fouad Nassar. Ses membres, d'origine palestinienne ou transjordanienne, ont participé à la guerre civile libanaise, auxcôtés du Fatah, ou du Front démocratique pour la libération de la Palestine (FDLP) de NayafHawatmeh.

${ }^{6}$ Pénélope Larzillière, La Jordanie contestataire. Militants islamistes, nationalistes et communistes, Arles, Sindbad/ Acte Sud, 2013, pp 43-58.

${ }^{7}$ Musa M. Shteiwi, «The Jordanian'sLeft : Today'sRealities and Future Prospects », in Jamil Hilal and Katja Herman (dir.), Mapping of the ArabLeft. ContemporaryLeftistsPolitics in the Arab East,Ramallah, Rosa Luxembourg StiftungRegional office in Palestine, 2014.
} 
unemême Coalition, des partis de gauche et nationalistes jordaniens (al-i'tilaf), les héritiers de Gamal Abdel Nasser et du b'athiste Michel Aflak ${ }^{8}$.

En somme, la gauche jordanienne est aujourd'hui plus réformiste que révolutionnaire.Surtout, elle est nationaliste. Mais c'est un nationalisme paradoxal,quiévolue selon des lignes de force tout à la fois jordaniennes, palestiniennes, et panarabes.Contrairement aux années 1960 et 1970, où la gauche jordanienne n'était que le reflet de ses alterego palestiniens, les formations de gauche jordaniennes se sont«jordanisées». Certes, la majorité des formations de gaucheportent encore la narration tiers-mondiste de la «libération de la Palestine ». Mais elles ont intégré la logique de l'État-nation dans leurs revendications notamment sociales. Pour certains groupes de gauche, c'est même une revendication nationaliste exclusivement jordanienne - voire hostile aux réfugiés palestiniens, idéalisant un modèle «tribal» transjordanien, en une figure toute khaldounienne du politique qui se dessine. La gauche jordanienne n'a cependant pas renoncé à un horizon d'attente transnational excédant les seules frontières de la Jordanie qui passe désormais plus par la Syrie que par la Palestine. La crise syrienneouverte en 2011 a réveillé les spectres nationalistes arabes — ou «machrékiens ${ }^{9} »($ mashriqiyya) — qui la taraudent encore. La gauche jordanienne symbolise, dans ses nationalismes en miroir, les succès d'un État-nation installé dans le temps et l'espérance de son audelà.

\section{La gauche jordanienne : un calendrier palestinien}

L'histoire de la gauche et des mouvements nationalistes arabes jordaniens est d'abord indissociable de la question palestinienne. Il faut attendre 1982 pour voir un Parti communiste palestinien (PCP) naître. Jusqu'à cette date, ses militants en Cisjordanie sont intégrés au Parti communiste jordanien (PCJ) - toléré par le pouvoir, il n'est cependant légalisé qu'en $1993^{10}$. Dans les années 1950, la distinction entre Jordanie et Cisjordanie est relative: le Parti de la renaissance arabe socialiste (Ba'th), qui demande sa légalisation auprès du gouvernement de Talal Al-Hechemi, après qu'eut

\footnotetext{
${ }^{8}$ La Coalition des partis de gauche et nationalistes jordaniens regroupe, entre autres, le Parti communiste jordanien (PCJ), Le Parti de l'unité populaire démocratique (Al-Wahda), le Parti du peuple démocratique jordanien (Al-Hashd), le Parti du renouveau arabe progressiste (branche du Baath pro-syrien), le Parti du renouveau arabe socialiste jordanien (branche du Baath pro-irakien), et des nassériens. Sur les partis politiques nationalistes et de gauche en Jordanie, voir Muhammad Jamal Barout (dir.), Al-ahzabwa-l-harakatwa-l-tandhimiyyat al-qawmiyya fi-l-watan al-'arabi[Les partis, mouvements et organisations nationalistes dans le monde arabe], Beyrouth, Markaz al-dirasat li-lwahda al-'arabiya, 2012, et Map of Political Parties and Movements in Jordan. 2013/2014, Netherlands Institute for MultipartyDemocracy, Embassy of the Kingdom of Netherlands in Jordan, 2014.

${ }^{9}$ Usuellement, le Machrek comprend la Palestine mandataire, la Jordanie, L'Irak, la Jordanie, la Syrie et le Liban.

${ }^{10}$ Jusqu'en 1943, les militants communistes de Cisjordanie sont intégrés à un Parti communiste palestinien, qui regroupe à l'époque Juifs et Arabes. Certains Palestiniens quittent alors l'organisation, et fondent une Ligue de libération nationale ('usbat al-taharrur al-watani), avant de rejoindre, en 1951, les rangs du PCJ.
} 
été édictée la Constitution de janvier 1952, est né un an plus tôt, à Ramallah, dans la maison d'Abdallah Al-Rimawi, son dirigeant.

Issu du Front démocratique pour la libération de la Palestine (FDLP), le Parti du peuple démocratique (Al-Hashd) ${ }^{11}$ est fondé en 1989, et légalisé en 1993. Et ce n'est qu'en février 1992 que les anciens activistes palestino-jordaniens du Front populaire pour la libération de la Palestine (FPLP) fondent le Parti de l'unité populaire démocratique (Al-Wahda). Les statuts fondateurs du parti coupent à peine le cordon ombilical avec la question palestinienne : leur deuxième chapitre appelle certes à construire une «société jordanienne socialiste». Mais la «libération de la Palestine» demeure «le but stratégique $»\left(\right.$ al-hadaf al-istratiji) ultime $^{12}$.

La gauche jordanienne et les nationalistes arabes sont, aujourd'huiencore, composés dans leur grande majorité de militants jordaniens d'origine palestinienne, et non transjordanienne ${ }^{13}$. La militance au quotidien est d'abord celle du calendrier politique palestinien: la Coalition des partis de gauche et nationalistes jordaniens célèbre successivement la Journée de la terre palestinienne (le 30mars) puis la Journée du prisonnier palestinien au mois d'avril. Elle organise des cérémonies de commémoration de la Nakba — l'exil palestinien de 1948 - dans les villes d'Amman, Zarqa, Irbid et Salt au mois de mai. Depuis octobre 2015, les cérémonies d'hommage aux «martyrs » palestiniens tombés lors de la «Troisième Intifada ${ }^{14}$ » se multiplient : le 5 mars 2016, la section de Zarqa d'Al-Wahda présente ses condoléances aux familles de Muhammad Zaghlul et de LabibKhaldoun, deux jeunes Palestiniens originaires de Qariout, près de la ville de Naplouse en Cisjordanie, tués par l'armée israélienne ${ }^{15}$.

L'imagerie palestinienne est omniprésente, et nourrit l'imaginaire militant : les locaux d'Al-Wahda sont saturés de portraits de Georges Habache, l'ancien fondateur du FPLP, longtemps militant du Mouvement des nationalistes arabes (MNA), tandis que ceux du Hashd affichent ceux de NayafHawatmeh ${ }^{16}$. Les campagnes contre la «normalisation» (tatbi') avec Israël sont continues. L'Association antisioniste et antiraciste (jam iyyamunahidasuhyuniyyawa'unsuriyya), fondée par un ancien

\footnotetext{
${ }^{11} \mathrm{Al}$-Hashd( la mobilisation) : acronyme de Hezb al-sha $\mathrm{b}$ al-dimukrati.

${ }^{12} \mathrm{Hizb}$ al-wahda al-sha'biyya al-dimukratiyya al-urduni,Al-barnamaj al-siyasi. Al-mu'atamar alwatani al-awwal[Le programme politique. La première conférence nationale], Amman, 1992, archives personnelles.

${ }^{13}$ Entretien avec FakhrDaas, membre de la direction d'Al-Wahda, Amman, février 2016.

${ }^{14} \mathrm{La}$ « Troisième Intifada » éclate en Cisjordanie et à Jérusalem-Est en octobre 2015, après que

les autorités israéliennes eurent interdit, un mois auparavant, les activités des Mourabitouns

(Gardiens), une association proche du Mouvement islamique palestinien (Branche Nord) du Cheikh Raed Salah.

${ }^{15}$ Azat Majid, «Wafd min al-wahda al-sha 'biyyayazurubayt 'aza al-shahidin Muhammad ZaghloulwaLalibKhaldoun » [Une délégation de l'Unité populaire visite la maison de deuil des martyrs Muhammad Zaghlul et LabibKhaldoun], Nida' al-Watan, 5 mars 2016, en ligne: http://wihdaparty.com/nashatat/54-jamahiriya/1621-2016-03-05-12-31-28

${ }^{16}$ Observations de l'auteur, Amman, février 2012.
} 
membre du Fatah résidant en Jordanie, IbrahimAlloush ${ }^{17}$, mène régulièrement des campagnes de boycott des produits israéliens.

Certains partis de gauche jordaniens sont enfin présents dans les camps de réfugiés palestiniens $^{18}$ : ils se substituent, en termes de représentation politique, aux partis politiques palestiniens, théoriquement interdits. La mise au ban de l'OLP en Jordanie date en effet de $1971^{19}$. En Jordanie, la direction des camps de réfugiés est dans les mains d'un Département des affaires palestiniennes (da'irat al-shu'un al-falistiniyya), dépendant du ministère des Affaires étrangères jordanien. Légalisée au début des années 1990, la branche jordanienne des Frères musulmans, proche du Hamas, a développé un réseau d'organisations nongouvernementales particulièrement bien implantées dans les camps, au détriment des associations proches du Fatah ou de la gauche palestinienne. Cependant, le Parti de l'unité populaire démocratique, proche du FPLP, et le Parti du peuple démocratique, issu du FDLP, conservent aujourd'hui des bureaux dans les principaux camps de réfugiés palestiniens.

\section{Un agenda national jordanien ?}

L'affinité élective de la majorité de la gauche jordanienne avec la question palestinienne est historique, et organique. Cependant, depuis la légalisation progressive de ces formations au début des années 1990, un agenda plus proprement national s'est imposé à elle. Les activistes privilégient le militantisme social et syndical, notamment au sein de l'Union des syndicats ouvriers indépendants(ittihad al-niqabat al-'umaliyya al-mustaqila), dans un contexte marquédepuis le milieu des années 2000 par une extension des conflits sociaux en Jordanie, notamment chez les travailleurs du port d'Aqaba (2009), les employés des mines, des métaux et du phosphate (2012), les travailleurs des impôts et les professeurs du secteur public $(2011)^{20}$. Le militantisme étudiant a repris une certaine vigueur depuis l'année 2007, date de fondation du mouvement Dhabahtuna («vous nous avez égorgés »), dont le porte-parole n'est autre que FakhrDaas, éditorialiste à l'hebdomadaire d'Al-Wahda, Nida ‘al-Watan («L’Appel de la Patrie »). En janvier 2016, Dhabahtuna organise des manifestations étudiantes dans le centre-ville d'Amman, pour protester contre l'augmentation des frais d'inscription dans les universités publiques jordaniennes ${ }^{21}$.

Depuis 2007, le Mouvement de la gauche sociale de Khaled Kalaldeh a tenté, avec des succès mitigés, de renouveler le socle intellectuel de la gauche jordanienne en

\footnotetext{
${ }^{17}$ Ibrahim Alloush est le fils de Naji Alloush, un important dirigeant du Fatah dans les années 1970, dont il était un des théoriciens de la branche de gauche.

${ }^{18}$ Treize camps de réfugiés palestiniens existent en Jordanie. Parmi eux, dix sont recensés comme des « camps officiels » par l'United Nations Relief and Works Agency for Palestine Refugees (UNRWA).

${ }^{19}$ En juillet 1971, le Fatah, ainsi que la majorité des organisations membres de l'OLP, retirent leurs combattants de Jordanie, après 10 mois de combats avec l'armée jordanienne.

${ }^{20}$ Entretien avec des représentants de l'Union des syndicats ouvriers indépendants, Amman, février 2016.

${ }^{21}$ Entretien avec FakhrDaas, membre de la direction d'Al-Wahda, Amman, février 2016.
} 
sensibilisant ses activistes aux thématiques altermondialistes, ou en intégrant, au sein d'une formation composée pour l'essentiel d'anciens membres du PCJ, des anarchistes jordaniens ${ }^{22}$ - sur le modèle des «nouvelles gauches », en Europe ou en Amérique latine, capitalisant sur le dialogue entre différences tendances idéologiques du spectre progressiste.

L'opposition,modérée, à la monarchie hachémite oblige également les mouvements de gauche à penser des stratégies d'alliances transversales - avec des courants n'appartenant pas au même univers idéologique. À partir de 1994, des formations comme Al-Wahda ou le Hashd se retrouvent dans des coalitions avec le Front d'action islamique (FAI), au sein du Haut comité de coordination des partis nationaux d'opposition $^{23}$. L'union avec les islamistes tient jusqu'en 2011 : elle se brise sur les réalités de la crise syrienne, le FAI prenant immédiatement parti pour l'insurrection contre Bashar Al-Assad. Si les formations de gauche et nationalistes arabes s'engagent enfin dans le mouvement social de janvier et février 2011, contestant la monarchie, certaines de ses figures dirigeantes intègrent le Haut comité de dialogue national mis en place en mars 2011 par le roi Abdallah : ainsi d'une dirigeante du Hashd, Abla Abu Ilbeh, ou du secrétaire général du Parti communiste, Mounir Hamarneh. La participation aux élections législatives de 2013 divise enfin la gauche jordanienne : si le Hashd et le PCJ s'engagent dans la course électorale, Al-Wahda s'en tient à sa traditionnelle politique de boycott des scrutins législatifs.

Question sociale, vie syndicale, élections, mouvements sociaux : un agenda proprement jordanien s'est imposé à la gauche et aux mouvements nationalistes arabes. Àtel point que, à partir de 2011, cette gauche voit naître un courant principalement intellectuel, mais pesant dans le débat public—se réclamant d'un nationalisme jordanien exclusif, si ce n'est chauvin : c'est-à-dire, pour l'essentiel, débarrassé de ses derniers oripeaux propalestiniens. Composé d'anciens membres du Parti communiste, ou du Mouvement de la gauche sociale, dans leur grande majorité transjordaniens, et non palestino-jordaniens, ce courant a son intellectuel organique : NahedHattar. Ancien communiste d'origine transjordanienne, c'est un intellectuel reconnu - mais contesté - dans le monde arabe. Il a longtemps été chroniqueur pour l'un des principaux quotidiens libanais, Al-Akhbar, de gauche et proche du Hezbollah. En septembre 2015, le journal libanais rompt cependant son contrat avec NahedHattar, lui reprochant des propos hostiles aux réfugiés syriens au Liban ${ }^{24}$.Cette mouvance se réfère parfois aux écrits d'un autre intellectuel jordanien résidant au Liban, enseignant à l'Université américaine de Beyrouth, TariqMoraiwed Tell, auteur

\footnotetext{
${ }^{22}$ Entretien avec MoazAbdelrahman et Moaz Abdo, anciens militants anarchistes du Mouvement de la gauche sociale, ayant quitté cette formation en 2013, Amman, février 2016.

${ }^{23}$ JilianSchwedleret Janine A. Clark, «Islamist-LeftistCooperation in the Arab World », ISIM Review, 1/18, 2006, pp. 10-11.

${ }^{24}$ Salah Dahun, «Khilafbayna NahedHattarwasahifatal-Akhbar li-inatihilaji'insuriyya » [Désaccord entre NahedHattar et le journal Al-Akhbar pour ses insultes envers les réfugiés syriens], Arabi21, 11 septembre 2015.
} 
d'un ouvrage de référence sur les origines sociales et économiques de la monarchie jordanienne $\mathrm{e}^{25}$.

Deux traits distinguent désormais cette gauche jordanienne de l'ancienne gauche issue du mouvement des fedayyin (combattants) palestiniens des années 1970. D’abord, la redéfinition d'un mouvement national jordanien endogène tel qu'il n'est pas déterminé, dans son histoire, par la seule narration propalestinienne d'une gauche supposément fille unique de la $\mathrm{Nakba}^{26}$, mais tirant ses origines des révoltes arabes contre Istanbul, à partir de juillet 1917, ou même des révoltes tribales de Karak contre les troupes de Sami Pasha Al-Faruqi, envoyé de l'Empire ottoman, lors de l'été $1910^{27}$. Ensuite, cette nouvelle gauche patriotique, dans son opposition au paradigme propalestinien, idéalise volontairement un sujet politique qui serait tout à la fois jordanien, nationaliste, nonpalestinien, et contestataire : à savoir le mouvement des tribus de l'estjordanien — particulièrement mobilisées contre la monarchie lors des journées de janvier et février 2011.

La mouvance de NahedHattar demeure minoritaire : elle s'attire qui plus est l'inimitié des Palestino-Jordaniens, qui lui reprochent, depuis 2011, de ne pas défendre les droits sociaux et politiques des réfugiés palestiniens en Jordanie ${ }^{28}$. NahedHattar a ses partisans, mais il n'est pas représenté par un courant politique particulier. Sa rhétorique n'en est pas moins symptomatique : la gauche palestinienne s'est bien jordanisée, nationalisée, au point d'avoir produit, en son sein, une tendance rompant radicalement avec la narration palestinienne qui lui avait donné naissance.

\section{Spectres nationalistes : arabes et machrékiens}

La gauche d'origine palestino-jordanienne n'a pas renoncé à la «libération de Jérusalem », mais elle se veut également jordanienne. NahedHattar dessine un nouvel imaginaire national, tout à la fois anti-néolibéral, patriotique et transjordanien. Les gauches jordaniennes ont fait le jeu de l'État-nation. Cependant, la crise syrienne, ouverte en mars 2011, a réveillé d'autres spectres nationalistes: arabes, ou machrékiens, ils contestent, à gauche, la seule formule d'un nationalisme jordanien réussi. C'est aussi que la Jordanie est partie prenante, de manière indirecte, au conflit :

\footnotetext{
${ }^{25}$ TariqMoraiwed Tell, The Social and EconomicOrigins of Monarchy in Jordan, New York, Palgrave Macmillan, 2013.

${ }^{26}$ Entretien avec Issam Hassan, ancien membre du Parti communiste jordanien proche de la mouvance de NahedHattar, et avec Issam Muhammad Al-Saadi, directeur du Centre machrékien pour les études géopolitiques, Amman, février 2016. Issam Muhammad Saadi est notamment l'auteur d'une histoire du mouvement national jordanien depuis 1921 :Al-Haraka al-wataniyya al-urduniyya. Al-kitab al-awwal (1921-1946). Muhawala li-i'dadkitabattarikh al-urdun[Le mouvement national jordanien. Tome 1 (1921-1946).Essai de réécriture de l'histoire de la Jordanie], Amman, Centre machrékien pour les études géopolitiques, 2010.

${ }^{27}$ Entretien avec NahedHattar, Amman, février 2016.

${ }^{28}$ Entretien avec des membres du Parti communiste jordanien, Amman, février 2016.
} 
elle a longtemps soutenu le Front du sud (al-jabha al-janubiyya $)^{29}$, proche de l'Armée syrienne libre (ASL), hostile à Bashar Al-Assad, avant de lui fermer, partiellement, ses portes en janvier 2016.

À gauche, la crise syrienne est une affaire classée, au moins idéologiquement : si le régime a besoin de réformes, le soutien à l'Armée arabe syrienne est entier. Elle est la dernière ligne de front contre une montée djihadiste perçue, de manière existentielle, comme l'ennemi principal. L'islam politique est la cible, mais à moitié seulement. Le Hezbollah conserve, dans la gauche jordanienne, un fort crédit ${ }^{30}$, à l'instar de l'Iran.

Les manifestations de soutien au régime syrien se sont multipliées depuis 2011 devant l'ambassade de Syrie à Amman, tout comme au centre de la capitale. La branche prosyrienne du parti Ba'th jordanien est membre de la Coalition des partis de gauche et nationalistes jordaniens. Le soutien au régime est devenu, depuis cinq ans, la nouvelle marque identitaire de la gauche. Plus que la question palestinienne, elle permet désormais de renouer avec un imaginaire nationaliste quin'est déjà plus le nationalisme jordanien. C'est d'abord un nationalisme arabe.

En février 2016, la Coalition des partis de gauche et nationalistes jordaniens organise, au siège d'Al-Wahda, à Amman, une cérémonie d'hommage (ta 'bin) à Rached Wadi Rahmi (Abu Kaid), décédé quelques mois plus tôt, en décembre $2015^{31}$. Né en 1953, c'est un ancien membre du Fatah de Yasser Arafat. Parti communiste jordanien, anciens de la gauche palestinienne, dirigeant d'Al-Wahda et du Hashd sont présents à cette cérémonie. En 1982, Rached Wadi Rahmiintègre les rangs du Fatah-Intifada scission de gauche du Fatah, emmenée par Abu Moussa qui s'allie rapidement au régime syrien. Rached Wadi Rahmiest un ancien des guerres du Liban. En 1991, il s'installe à Amman. Depuis 2013, il participait aux activités militaires de la Garde nationaliste arabe (GNA), une petite formation militaire, d'orientation nationaliste arabe, constituée de quelque deux mille combattants d'origine algérienne, égyptienne, jordanienne, tunisienne et palestinienne, présente dans la banlieue de Damas ${ }^{32}$. Elle se bat, au nom d'une idéologie tout à la fois de gauche et nationaliste arabe, auxcôtés des troupes du régime syrien.

Le court documentaire sur la vie de Rached Wadi Rahmi, diffusé à l'attention de la centaine de participants, comprend un hommage de la GNA à cet ancien membre du Fatah. Des hommes masqués, en armes, défilant dans les rues de Damas avec son portrait. Une fois le documentaire terminé, le secrétaire général d'Al-Wahda,

\footnotetext{
${ }^{29}$ International Crisis Group, « New Approach in SouthernSyria », Middle East Report163, septembre 2015.

${ }^{30}$ Entretien avec des activistes d'Al-Wahda, Amman, février 2016. Voir également l'article de NahedHattar sur le chiisme dans le monde arabe : «Al-nahda al-'arabiya : al-marhala al-shi'iyya » [La renaissance arabe : l'étape chiite], Al-Akhbar, 23 juin 2015.

${ }^{31}$ Observations à l'invitation d'Al-Wahda, Amman, février 2016.

${ }^{32}$ Nicolas Dot-Pouillard, «La gauche arabe : orpheline de révolution », Revue Moyen-Orient, janvier 2016.
} 
SaidDhiab, prend la parole : il rend hommage d'abord aux «martyrs » palestiniens du FPLP, du Hamas et du Mouvement du Jihad islamique en Palestine (MJIP). Et conclut sur le rôle de l'Armée arabe syrienne dans la "résistance au projet saoudien et takfiri». De la Palestine à la Syrie, la gauche jordanienne a retrouvé ses accents nationalistes arabes. C'est, au moins depuis la fondation du Mouvement des nationalistes arabes ${ }^{33}$ dans les années 1950, une partie constitutive de son ADN idéologique — autant que le marxisme.

Le nationalisme arabe de la gauche jordanienne a trouvé une seconde vie à Damas sans doute plus qu'à Ramallah ou à Gaza. Pour les partisans de gauche de NahedHattar, c'est moins le nationalisme arabe qui est célébré, qu'un nationalisme appelant à l'unification — sous forme fédérale — du Machrek. Il s'agit de prendre appui sur la guerre en Syrie pour mobiliser un imaginaire transnational alternatif à celui des islamistes. Le 17 janvier 2016, l'intellectuel jordanien organise une conférence à Beyrouth, après plusieurs séances préparatoires à Damas. La Rencontre consultative machrékienne (al-liqa' al-tashawuri al-mashriqi) est officiellement lancée. Son communiqué fondateur débute parun hommage appuyé à «l'Armée arabe syrienne et ses partisans dans leur combat contre l'agression impérialiste, réactionnaire, ottomane et terroriste sur la République arabe syrienne $»^{34}$. L'islam politique - seulement sunnite- et la Turquie sont des cibles évidentes. Ce nationalisme machrékien prend également appui sur le réveil, en Jordanie, du Parti syrien national social (PSNS). Né en 1932, au Liban, fondé par AntunSaade, il n'a qu'un rapport lointain avec les gauches arabes. Il fut même anticommuniste. La branche jordanienne du PSNS milite aujourd'hui auxcôtés de la Coalition des partis de gauche et nationalistes jordaniens. Le PSNS a ses milices en Syrie, et ses assises politiques au Liban ${ }^{35}$. Son projet pan-national, visant à réunifier une Grande Syrie mythifiée et «laïque », courant de la Palestine historique au Liban, en passant par Damas, fascine parfois à la gauche du spectre politique ${ }^{36}$.

\section{Penser les nationalismes}

La gauche jordanienne est un idéal-type des gauches arabes : sa longue histoire pose moins de questions sur le marxisme, l'anarchisme, la social-démocratie, l'extrême et le centre-gauche, que sur la question nationale et sa centralité. La gauche

\footnotetext{
${ }^{33}$ Le Mouvement des nationalistes arabes (MNA) a notamment donné naissance au Front populaire pour la libération de la Palestine (FPLP) et à l'Organisation d'action communiste au Liban (OACL), à la fin des années 1960.

34 «Al-liqa' al-tashawwuri : al-Qararat al-siyasiyya» [Rencontre consultative : les décisions politiques], Beyrouth, 17 janvier 2016, archives personnelles.

${ }_{35}^{35}$ Nicolas Dot-Pouillard, «Sur les frontières : le Parti syrien national social entre idéologies unitaires et États-Nations », in Pierre-Jean Luizard et Anna Bozzo (dir.), Les printemps arabes cinq ans après : États et sociétés civiles face aux logiques segmentaires, Rome, Roma Tre, (à paraître).

${ }^{36}$ Ainsi de plusieurs anciens militants du Mouvement de la gauche sociale et du Parti communiste libanais, d'une moyenne d'âge d'une trentaine d'années, que j'ai interviewés en février 2016. Si leur socle idéologique est marxiste, les références sont aussi hétéroclites: Parti syrien national social, Hezbollah, Iran, expériences nationalistes de gauche latino-américaines de type «chavistes ».
} 
jordaniennec'est, à l'origine, une histoire palestinienne. Lorsqu'elle reconnait, au détour des années 1990, la logique de l'État-nation jordanien, elle ne le fait qu'à demi-mot. Il y a le nationalisme, et ses doubles. Nationalismes arabes ou machrékiens, tous deux spectres réveillés par la crise syrienne : l'articulation entre un nationalisme jordanien et d'autres types de nationalisme demeure difficile. Le Parti $\mathrm{Ba}$ 'th tenta d'y répondre, à une époque lointaine, par une articulation conceptuelle complexe entre les dimensions nationalistes arabes (qawmiyya) et ses différentes articulations locales (qutriyya). Les Partis communistes, tout comme les «nouvelles gauches » des années 1960 et 1970, développèrent parfois l'idée d'une fédération arabe socialiste - tout à la fois reconnaissante de l'État-nation, mais pensant son audelà.

Entre utopies transnationales et acceptation de l'État-nation, la gauche jordanienne a encore ses horizons d'attente conflictuels. Elle s'est indéniablement nationalisée. Composante de l'opposition démocratique, un temps alliée au Front d'action islamique, elle a son agenda jordanien: il est social et syndical. C'est plus particulièrement vrai pour la Coalition des partis de gauche et nationalistes. Les théories nationalistes jordaniennes de NahedHattar, s'appuyant sur une idéalisation des «tribus » de l'Est jordanien comme nouveau sujet du politique, tout comme le rapprochement de certaines composantes de la gauche avec le gouvernement, à l'instar de Khaled Kalaldeh et du Mouvement de la gauche sociale, sont un symptôme : la greffe de l'État-nation a prise. La gauche a son calendrier jordanien. Elle préserve néanmoins son agenda transnational : il est tourné vers Jérusalem, mais regarde aussi vers Damas.

Qui gagne, de la logique de l'État-nation ou des horizons d'attente transnationaux ? L'erreur consisterait à vouloir trancher. La gauche jordanienne n'est pas la seule à vivre ce dilemme. Ses utopies transnationales, de Jérusalem à Damas,permettent de penser la question nationale dans le monde arabe en la comparant à d'autres courants : maintenant opposée aux Frères musulmans, notamment sur la crise syrienne, la gauche jordanienne n'en partage pas moins avec le Front d'action islamique un élément commun. Les islamistes jordaniens se sont en effet «jordanisés » et nationalisés, à l'instar d'autres partis islamistes dans le monde arabe. Pourtant, ils n'ont en rien renoncé à un au-delà de l'État-nation — leur soutien forcené à l'opposition syrienne, ou au Hamas palestinien, en témoigne. Peut-être serait-il plus heuristique de comparer la gauche et les islamistes dans leurs rapports contrariés à la question nationale, que de les opposer sur des affaires de conjoncture politique : ils partagent au final la même valse hésitation sur l'État-nation et son devenir. 